\title{
THEORETICAL-EXPERIMENTAL POSSIBILLITIES OF MICROSTRUCTURE QUANTIFICATION OF DISPERSION STRENGTHENED MATERIALS
}

\author{
Michal Besterci ${ }^{1)^{*}}$, Katarína Sülleiová ${ }^{1)}$ \\ 1) Institute of Materials Research of Slovak Academy of Sciences, Košice, Slovak Republic
}

Received: 14.12 .2018

Accepted: 28.02.2019

${ }^{*}$ Corresponding author: e-mail: mbesterci@saske.sk Tel.: +421 55 692241, Institute of Materials Research of Slovak Academy of Sciences, Košice, Watsonova 47, 04001 Košice, Slovak Republic

\begin{abstract}
The present paper is devoted to the possibilities to classify the spatial arrangement of the elements (features) of a stochastic process with geometrical objects. The fundamental quantities describing point processes were introduced. Experimental possibilities of structural objects determination, possibilities of evaluation of the size distribution of the secondary phases, testing of planar point structures (estimation of the process intensity, square method and characteristics of the second order) were estimated. Interparticle distances, namely mean interparticle distance, mean minimum distance, mean visibility and mean path of spherical contact were defined. Selected processes were described and demonstrated from simulated realizations on $\mathrm{Al}-\mathrm{Al}_{4} \mathrm{C}_{3}$ dispersion strengthened material, prepared by a powder metallurgy method of reaction milling. Interparticle distances of $\mathrm{Al}_{4} \mathrm{C}_{3}$ particles were evaluated. Polygonal methods and quadrant counts method were used for characterization of the particle arrangement.
\end{abstract}

Keywords: mechanical alloying, structure parameters, interparticle distance, testing of planar point structure

\section{Introduction}

Intensive research and development of new materials and their challenging technologies belong among those matters which become determining for the recent worldwide industrial and economical progress. They lead to a considerable diversification of materials which makes the choice of the optimal material for any considered application possible.

The research proceeds in several directions the most important of which are new metallic alloys, ceramics, polymers, composites and amorphous materials. The composite materials with metallic, plastic and ceramic bases have already found their application in the machine industry, but their wider use is still limited by insufficient automation on their production resulting in their cost and by the need to elaborate new designs and techniques adapted for a better understanding and influencing of their properties, [1-8].

The mechanism of dispersion strengthening is based on the presence of a low volume fraction of fine secondary particles in the metal matrix, [9 - 11]. If the particle sizes are negligible, with respect to their mutual distances, they can be considered as points. Particles in real material can be visualized in the cross section of the microstructure by optical microscopy and scanning electron microscopy (SEM) or in a projection by transmission electron microscopy (TEM). Quantitative characterization of the particle space distribution is a significant problem, [12- 14]. Classification of the $\mathrm{Al}_{4} \mathrm{C}_{3}$ particle arrangement in $\mathrm{Al}_{-} \mathrm{Al}_{4} \mathrm{C}_{3}$ materials with 2 wt. $\% \mathrm{Al}_{4} \mathrm{C}_{3}$ using the stochastic geometry method was the aim of the paper. 


\section{Experimental possibilities of determination of structural objects}

Experimental determination of the basic structural parameters of the materials strengthened by particles is very important and demanding from the experimental point of view. Therefore, some situations and principal possibilities of obtaining the information mainly on the volume fraction of dispersion particles and on their size distribution were analyzed. Let us assume further the spherical particles distributed in space in the uniform stochastic manner. Let us to define the total volume fraction $f_{v}$. The particle size is defined by the probability density function $h(d)$.

\section{a) Plane metallographic section through the structure}

The plane section through the structure in question represents the two-dimensional space with circular particles; their volume fraction is $f_{A}$ and the probability density function of the particle sizes in the plane is $g(d)$. In this case, the well-known stereological relationship $f_{v}=f_{A}$ is valid.

Moreover, it is possible to prove that $\lambda_{3}=\lambda_{2}$ [15], which allows theoretically the direct experimental determination of the mean interparticle distance $\lambda$. The distance $\left(\Lambda_{\min }\right)$ cannot be determined from the plane section for general distribution of the particles in space. For monodispersion systems, it is possible to find the relation between the planar and volume density of particles, and to determine the distance $\Lambda_{3}$ on the basis of the known value of the planar density. However, for polydispersion systems, it is necessary to know the shape of the function $h(d)$ on the basis of plane sections, in order to determine the mean particle distance $\Lambda_{3}$. This task can be solved, and is discussed in the paper [16]. On the basis of the plane sections, it is also possible to determine other individual and global characteristics of particles.

The sizes of particles of the dispersion strengthened systems, which were prepared by internal oxidation, or by chemical precipitation, ranged below $100 \mathrm{~nm}$. The sizes of the effective particles $(\sim 70 \%)$ of the systems, which were prepared by reaction milling, were below $200 \mathrm{~nm}$. The above described methods, which give sufficient information, cannot be used for the resolution of an optical microscope, nor can be used for the whole range of granulometric composition of the particles, They can be used only for estimation of the largest particles, which do not have the decisive influence on mechanical properties.

\section{b) Extraction carbon replicas}

Contrary to the plane metallographical section, a structure, in which the ,two-dimensional particles" do not represent the plane sections through the three-dimensional particles, but only the projections of particles to a plane, was studied. The original Asby-Eberling model [17] assumes that all particles, which have the intersection with the plane (the depth of matrix etching below this plane is larger than the maximum size of the particles), are included in the replica. In this case, the probability density function of projections of particles $g(d)$ can be determined experimentally. The following relationship is valid between the functions $g(d)$ and $h(d)$ :

$g(d)=\frac{d h(d)}{\int_{0}^{\infty} d h(d) d d}$

which allows the shape of the function $h(d)$ to be determined.

It is then possible, on the basis of the planar density of particles and using function $h(d)$, to determine the volume density of particles and the distances $\lambda_{3}$, or $\Lambda_{3}$, respectively. If the etching depth is smaller than $d_{\max }$, some of the particles will not be ,trapped" in the replica, but a print will be formed. This case has been analyzed by Horalek [18]. Further information is then necessary to determine the form of the function $h(d)$ (the depth of etching, general form of the function $h(d)$, the number of particles, which are not included in the replica, but only printed).

Moreover, the basic model is idealized. In the real material, the situation is complicated, both by non-uniform etching and by the presence of the accumulated particles. If the clusters of 
accumulated particles are present on the replicas, then we can use them for rough estimation of the form of the function $h(d)$ in such a way that they are considered to be the random sampling from the set of particles. In addition, the accumulated particles can serve as the low limit of estimation of the etching depth.

\section{c) Thin foil}

This case is the simplest one from the theoretical point of view. Projections of the particles in a plane by examination of the foil by the electron beam were obtained. The function $h(d)$ can be determined directly on the basis of the assumptions of the particle shape. However, in real experiments, the problem of measuring the thickness of the foil at the examined point arises. In addition, there is a problem of behavior of the particles in the surface layers of the foil under reduction of its thickness, as well as the problem of overlapping particles. These problems can be partially solved using a goniometer and a scanning adapter on a transmission electron microscope. In spite of the above facts, it is necessary to regard the thin foil as the most reliable source of information, mainly on the finest fractions of the dispersion phase.

\section{Models of heterogeneous structures and their evaluation}

The conventional parameters, such as volume fraction and interparticle distance, do not depend on the spatial arrangement of the particles and should be replaced by other variables, which are sensitive to the structure. These include various types of stochastic free paths, at which more direct physical interpretation is assumed in connection with the transport processes. From this point of view, various models of the spatial arrangement of particles (stochastic closed sets, combined sets of particles with the given probable distribution of the shape, size and positions) are analyzed in the monograph [19]. The results include stochastic systems, which do not influence each other, stochastic clusters, homogeneous systems of particles, homogeneous systems with defects (vacancies, interstices, pores, foreign particles) and they are documented by numerous diagrams. General solution of this problem allows the results to be applied to the linear, planar and spatial systems, respectively.

\subsection{Possibilities of evaluation of the secondary phases distribution}

The mechanism of dispersion strengthening is based on the presence of a relatively small quantity of fine particles in the matrix. If the size of these particles is substantially smaller than their distances, they can be considered, in the first approach, as the "point" objects from the viewpoint of their distribution. Their distribution in the matrix can play an important role at their effects on the process taking place in the matrix.

It is necessary to realize that the particles of certain sizes and shapes are arranged in space in the real dispersion strengthened material. In order to make them visible, a section through the microstructure can be made (metallography or SEM), or to depict them in their projection using transmission electron microscopy (TEM). The signs of the spatial distribution of the real particles of sizes different from zero are manifested in both the section and in the projection, but the signs of the distribution of the point particles are manifested only in the projection. The quantitative characterization of the spatial distribution of particles is very important task. It was used only sporadically in physical metallurgy. The basic stochastic models of the spatial distribution of particles are as follows:

a) The Poisson point process, which serves as a reference of stochastic distribution of points, is defined as the position of an arbitrary point, which does not depend on the position of other points. 
b) The hard-core model, for which the condition requiring that the distance of two arbitrary points of the process is larger than the value determined in advance.

c) The cluster model, which can be created on the basis of the Poisson point process in such a way that each point of the system is replaced by a cluster of points.

d) The grid arrangement of points with defects, such as interstices, vacancies and displacement of points from their equilibrium position.

The following two methods are often used for evaluation of the spatial distribution of particles. With the first method, the point planar processes are tested, the second one is based on the term of the interparticle distance.

\subsection{Testing of planar point structures}

\section{a) Estimation of process intensity}

The question is about the estimation of the number of points in the unit of the area studied assuming the homogeneous structure. Accuracy of the standard used procedure (the ratio between the number of points and the surface measured) depends strongly on the type of points distribution. For the usually used level of estimation (95\%) about 1600 points are needed in the area to obtain the ideal stochastic distribution (Poisson point process). If a large number of clusters of points are present, the number of objects must be considerably greater in order to obtain the above accuracy [20].

\section{b) Square method}

The area studied is divided into a large number of subareas of the same size, in which the number of points is ascertained. On the basis of the mean number of points and their dispersion in these subareas, various statistics are prepared (index of dispersion, cluster size index, Greig-Smith test and others), which can indicate a tendency of points towards clustering, or towards regular arrangement respectively. It is necessary to realize for all indices that their value depends not only on the distribution of points, but on the size of subareas as well. Therefore, it is recommended to evaluate also the dependence of the index on the size of the subareas.

\section{c) Characteristics of the second order}

These characteristics describe in detail the spatial distribution of points. They can be determined using, for example, the K-function $K(r)$, whose course is given by the dependence between the number of points in the circular surrounding $r$ of the typical process point on the size of $r$. The $L$ function is also used, which is defined by the relationship $L(r)=(K(r) / \pi)^{1 / 2}$, and the pair correlation function $g(r)$, which characterizes the change rate $K(r)$ in dependence on $r, g(r)=(2 \pi r)^{-1 / 2} d k / d r$; for the planar Poisson point process, the following is valid: $K(r)=\pi^{2}, g(r)=1$ and $L(r)=r$.

Information on the spatial distribution of particles in relation to their sizes and on the spatial distribution of the volume fraction are then obtained.

\subsection{Interparticle distances}

Problems of the definition of the interparticle distances both for the monodispersion and polydispersion set of the spherical particles were discussed in the previous section. One of the conclusions of the papers says that the only parameter (mean value) used to characterize the granulometric composition of the dispersoid in the real polydispersion systems can distort the theoretical estimation of the interparticle distance of the type $\lambda_{\mu}$. When one uses the term "interparticle distance", it is necessary to realize, (e.g. for characterization of materials or processes which take place in them) that this term is rather intuitive, and has no generally valid, 
universal definition. Therefore, it cannot be assigned the generally valid range of values. At first, we must select one of the exactly defined types of "distances" considering the process studied and to interpret or compare their properties within the selected framework.

a) Mean interparticle distance $\lambda_{\mu}$

This is the mean value of chord lengths of the generated systems of isotropic uniform stochastic straight lines intersecting the object studied. It is often referred to as the "mean free path" between the particles. Its "positive" property is that after observing the condition of stochasticity of the system of plane sections through the volume structure, it is possible to prove that $\left(\lambda_{\mu}\right)_{3}=\left(\lambda_{\mu}\right)_{2}$ (i.e. $\lambda_{\mu}$ of the sample equals $\lambda_{\mu}$ of its isotropic, uniformly stochastic plane sections), which has contributed to its frequent use. However, its negative property is that it does not depend on the spatial distribution of the particles, because it is a global variable, which is fully determined by the total volume and surface of the sample particles.

\section{b) Mean minimum distance $\lambda_{p}$}

This is the mean value of the distance between the nearest particles. Its advantage is based on the fact that it is well worked out for the Poisson stochastic process, however, the mechanical use of these theoretically derived relations for any other structure is confusing. It is necessary to state that this parameter describes well the distance within the particle clusters, however, it has no informative ability, therefore its physical and metallurgical interpretation is problematic.

c) Mean visibility $\lambda_{v}$

This is the mean value of the distance from the randomly selected point of the matrix to the nearest particle in the randomly selected direction. This distance can be described as the "mean visibility in a forest" (for the plane case). Its properties, mainly the relation between the distance in the volume and the respective plane section, have not been theoretically worked out yet. Physical and metallurgical interpretations in relation to the obstacles for dislocation movements are also problematic.

\section{d) Mean path of spherical contact $\lambda_{v}$}

This is the mean value of the distance from the randomly selected point of the matrix to the nearest particle. It determines the distribution of sizes of the maximum spheres inscribed into the matrix in its internal points; therefore, it depends on the spatial distribution of particles [20]. It has suitable limit properties, and can be easily measured manually. From the physical and metallurgical viewpoint, it can be interpreted, for example, as the characteristic of the minimum diameters of dislocation loops of the emitted F-R sources. Therefore, we consider it to be the optimum distance of particles in the dispersion strengthened systems.

However, it is necessary to state that at the quantification of the heterogeneous systems with particles, the mean distance, which was determined as the cube root of the average volume per one particle, was frequently used. It is determined by the number of particles in the volume unit and does not depend on the spatial distribution of particles. The differences in the values of the interparticle distances defined in such a way are documented in Fig. 1, where their mean values are plotted in the simulated Boolean model of spherical particles (the Boolean model is created by substitutions of models of particles with various shapes for individual points of any point process). In our previous works $[19,21,22]$ the distances between the particles by point object simulation methods were evaluated. This included the mean interparticle distance $\lambda_{\mu}$, the mean minimum distance $\lambda_{\rho}$, the mean visibility $\lambda_{v}$, and the mean free spherical contact distance $\lambda_{\vartheta}$. The characteristics and properties of these parameters have been analyzed in [21]. The mean free spherical contact distance $\lambda_{\vartheta}$ was the optimal parameter which had a physical interpretation, too. 
During the last years, a new approach to the description of point systems has been developed intensively, which was referred to as polygonal methods [22]. The composite system $\mathrm{Al}_{-} \mathrm{Al}_{4} \mathrm{C}_{3}$ prepared by powder metallurgy was used for the experiments. The starting powders $\mathrm{Al}$ (of size < $100 \mu \mathrm{m})$ and $\mathrm{C}(2$ wt. \%) were dry mechanically alloyed for 90 minutes, compacted under the pressure of $600 \mathrm{MPa}$ (rod diameter $40 \mathrm{~mm}$ ), annealed at $550^{\circ} \mathrm{C} / 3 \mathrm{hr}$ and then extruded with $94 \%$

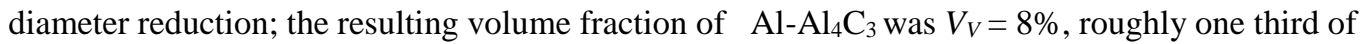
$V_{V}$ was comprised in very coarse particles. Thin foils if the thickness $\mathrm{L}=(2000 \pm 500) \AA$ were prepared by spark cutting followed by electropolishing and did not include coarse particles.

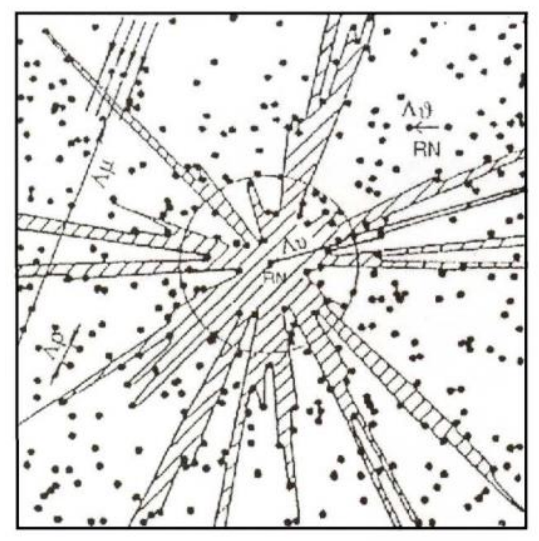

Fig. 1 Schematic illustration of interparticle distances

Properties of Voronoi tessellation and their various generalizations were studied in the monograph [23].The dual representation formed in the above way described completely the given point system. Intermediate stages of evaluation for thin foil (a), outlines of particles (b), and of reference points (c) were documented in Fig. 2.
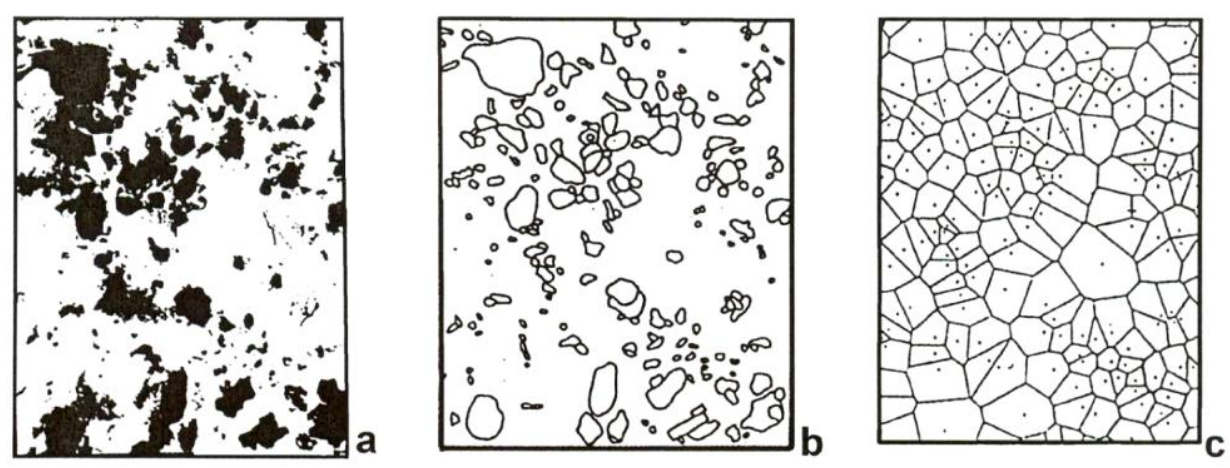

Fig. 2 Intermediate stages of evaluation for thin foil (a), outlines of particles (b) and reference points (c)

The practice of the polygonal methods in describing the spatial arrangement of particles is explained in detail, namely momentum analysis, order statistics and parametric estimation of the 
probabilistic distribution, all in the relation to the cell areas of the Voronoi tessellation generated by the reference points of the particles. The polygonal approach is then applied to four samples, Fig. 3 - TEM projections of thin foils of an aluminium based dispersed alloy with quasiglobular particles. The main advantages of the method are stressed: reliable characteristics of the arrangement can be obtained on the basis of relatively small samples and without the accurate knowledge of the thin foil thickness. Moreover, the sensitivity is higher in comparison with more standard approaches (e.g. quadrant counts), [24 - 28].

a
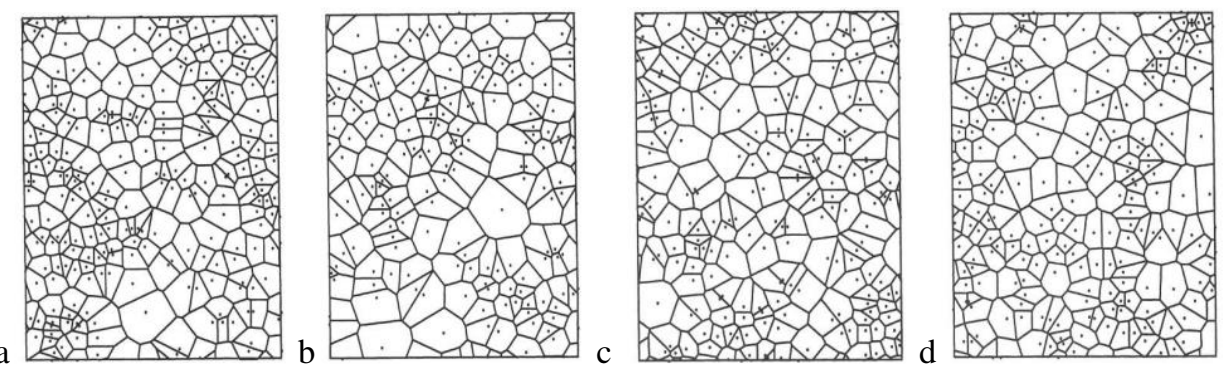

Fig. 3 Voronoi mosaics generated by point patterns (a), (b), (c) and (d)

\section{Conclusions}

General problems of testing the structure of materials produced by PM technology were summarized as well as possibilities of the quantitative estimation of structure parameters and basic problems connected with the experimental techniques:

1. Models of heterogeneous structures and approaches of quantitative evaluation of secondary phases by stereological methods (squares method, dual method by means of Voronoi mosaics) were defined.

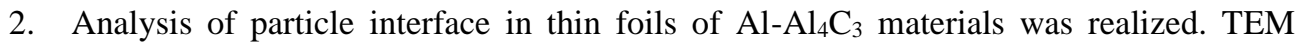
images of thin foils with quasi-globular particles were examined by means of two methods of spatial statistics. The spatial arrangement of particle reference points was described by means of quadrate count statistics and by polygonal method (the analysis of the Voronoi mosaic generated by patterns of particle reference points). A good agreement between the both approaches was found, the polygonal method was more sensitive and its results were more conclusive.

3. Six important types of interparticle distances based mainly on the concept of matrix free paths or rays were introduced. The distribution functions and probability density functions of the path and ray lengths as well as the expressions for the first two moments of these distributions were deduced for the Poisson point process and Boolean model.

\section{References}

[1] T. Kvačkaj et al.: Materials Characterization, Vol.134, 2017, p. 246-252, DOI: 10.1016/j.matchar.2017.10.030

[2] T. Kvačkaj et al.: Archives of Metallurgy and Materials, Vol. 58, 2013, No. 2, p. 407-412, DOI: 10.2478/amm-2013-0008

[3] D. Manfredi, R. Bidulský: Acta Metallurgica Slovaca, Vol. 23, 2017, No. 3, p. 276-282, DOI: $10.12776 /$ ams.v23i3.988

[4] P. Petroušek et al.: Acta Metallurgica Slovaca, Vol. 21, 2015, No. 3, p. 176-183, DOI: 10.12776/ams.v21i3.615 
[5] T. Kvačkaj, R. Kočiško, J. Bidulská, M. Luptak, A. Kováčová: Acta Physica Polonica A, Vol. 131, 2017, No. 5, p. 1315-1318, DOI: 10.12693/APhysPolA.131.1315

[6] R. Bidulský, M. Actis , M. Kabátová, J. Bidulská: Journal of Materials Science \& Technology, Vol. 25, 2009, No. 5, p. 607-610

[7] A. Fedorikova et al.: Acta Metallurgica Slovaca, Vol. 22, 2016, No. 2, p. 102-110, DOI: $10.12776 /$ ams.v22i2.616

[8] R. Bidulský, M. Actis , L. Ferraris, P. Ferraris, J. Bidulská: Acta Physica Polonica A, Vol. 118, 2010, No. 5, p. 802-803, DOI: 10.12693/APhysPolA.118.802

[9] I. Saxl: Stereology of Objects with Internal Structure, Elsevier, ISBN 0444416854, 1989

[10] M. Besterci: Dispersion Strengthened Al Prepared by Mechanical Alloying, Cambridge Int. Sci. Publ., 1999, ISBN 189832655

[11] T. Weißgäerber, B. Kieback: Materials Science Forum, Vol. 343, 2000, p. 275-283, DOI: 10.4028/www.scientific.net/MSF.343-346.275

[12] J. Bidulská, T. Kvačkaj, I. Pokorny, R. Bidulský, M. Actis Grande: Archives of Metallurgy and Materials, Vol. 58, 2013, No. 2, p. 371-375, DOI: 10.2478/amm-2013-0002

[13] J. Bidulská, T. Kvačkaj, R. Bidulský, M. Actis Grande, L. Litynska-Dobrzynska, J. Dutkiewicz: Chemicke Listy, Vol. 105, 2011, p. s471-s473

[14] J. Bidulská, T. Kvačkaj, R. Bidulský, M. Actis Grande: Acta Physica Polonica A, Vol. 122, 2012, No. 3, p. 553-556, DOI: 10.12693/APhysPolA.122.553

[15] M. Besterci, K. Pelikán: Kovové materiály, Vol. 4, 1985, p. 497-501

[16] E. E. Underwood: Quantitative Stereology, Addison-Wesley Publ. Co., Reading, Massachusetts, 1970

[17] M.F. Ashby, R. Eberling: Transactions of the Metallurgical Society of AIME, Vol. 236, 1966, p. 1396-1401

[18] J. Horalek: Kovové Materiály, 3, 1982, p. 195

[19] I. Saxl, K. Pelikán, J. Rataj, M. Besterci: Quantification and Modelling of Heterogeneous Systems. Cambridge Interscience Publ., ISBN 1898326045, 1995

[20] I. Saxl, K. Pelikán, M. Besterci, J. Rataj: Testing and Modelling Structures of Heterogeneous Systems, Pokroky práškové metalurgie, Vol. 1-2, Šumperk, 1990

[21] I. Saxl, M. Besterci, K. Pelikán: Pokroky práškové metalurgie , Vol. 3, 1986

[22] M. Besterci, I. Kohútek, I. Saxl, K. Sülleiová: Journal of Materials Science, Vol. 34, 1999, p. 1055-1060, DOI: 10.1023/A:1004552213455

[23] A. Okabe, B. Boots, K. Sugihara: Spatial Tessellations: Concepts and Applications of Voronoi Diagrams, J. Wiley, Chichester (1992), ISBN: 0-471-9340-5

[24] M. Besterci, I. Kohútek, I. Saxl, K. Sülleiová: Kovové materiály, Vol. 33, 1995, No. 4, p. 251-268

[25] M. Besterci, J. Zrník, M. Šlesár: Kovové materiály, Vol. 35, 1997, p. 344-349

[26] M. Besterci, O. Velgosová, J. Ivan, L. Kováč: Kovové materiály, Vol. 39, 2001, p. 309-315

[27] M. Besterci, O. Velgosová, I. Kohútek: Iranian Journal of Science \& Technology, Transaction B, Vol. 28, 2002, No.B2, p. 233-238

[28] M. Besterci et al.: Nanostructural Al Composites, Lambert Academic Publishing, 2015, ISBN-10: 3659773875

\section{Acknowledgements}

The work was supported by the Slovak National Grant Agency under the Project VEGA 2/0080/17. 